\title{
Demenz in der oralen Medizin: Erkennen von Zeichen der Erkrankung - Einschätzen von Ressourcen
}

\author{
Christian E. Besimo Ruth H. Besimo-Meyer \\ Orale Medizin, Seeklinik, Brunnen, Schweiz
}

\section{Schlüsselwörter}

Demenz Patientenscreening in der oralen Medizin .

Symptomerkennung $\cdot$ Ressourceneinschätzung

\section{Zusammenfassung}

Die zunehmende Zahl von an Demenz erkrankten Menschen stellt auch die orale Medizin vor erhebliche Herausforderungen. Über $60 \%$ der von Demenz Betroffenen leben ohne Diagnose und erhalten somit weder eine adäquate medizinische noch eine oralmedizinische Betreuung. Somit sehen sich die Zahnärzte vor die Notwendigkeit gestellt, selbst kognitive Defizite bei ihren älteren Patienten erkennen zu können, um Fehlentscheidungen bezüglich Diagnostik, Prävention und Therapie zu vermeiden. Demenzkranke erfordern aber auch besondere Fähigkeiten in der Kommunikation. Die vorliegende Arbeit stellt deshalb Instrumente vor, die im oralmedizinischen Kontext geeignet sind, um kognitive Defizite zuverlässiger erkennen, verbleibende Ressourcen besser einzuschätzen und die Kommunikation optimieren zu können.

(c) 2016 S. Karger GmbH, Freiburg

\section{Keywords}

Dementia Screening of patients in oral medicine . Recognition of symptoms - Estimation of resources

\section{Summary}

Dementia in Oral Medicine: Detecting Signs of the Disease -

\section{Estimating Resources}

The increasing number of people suffering from dementia poses a major challenge also in oral medicine. More than $60 \%$ of persons suffering from dementia live without a confirmed diagnosis and thus receive neither adequate medical nor oral health care. Thus, the dentists themselves are faced with the need of being able to detect cognitive deficits in their older patients to avoid incorrect decisions regarding diagnosis, prevention, and therapy. However, patients with dementia also require special skills in communication. This paper therefore introduces adequate instruments in oral health care which help to detect cognitive deficits more efficiently, to better estimate remaining resources, and to optimize communication.

menz ziehen sich in eine Welt zurück, in der Dinge und Ereignisse eine andere Bedeutung erhalten und die demzufolge nur noch schwer erreichbar ist. Die Kommunikation wird zunehmend schwieriger, insbesondere bei Verlust des Sprachvermögens. Zum Ende geht nicht nur die geistige Leistungsfähigkeit weitestgehend verloren, sondern es kann zusätzlich infolge von Multimorbidität eine deutliche körperliche Beeinträchtigung hinzutreten $[1,2]$.

\section{Fehlende Diagnosestellung und interdisziplinäre Vernetzung}

In der Schweiz waren 2010 etwa 110000 Menschen von Demenz betroffen - mit einer Inzidenz von etwa 26000

\section{KARGER \\ Fax +497614520714}


Personen pro Jahr. Man geht heute davon aus, dass über 60\% der an Demenz erkrankten Menschen ohne Diagnose leben und somit zu rund $75 \%$ von keiner adäquaten medikamentösen bzw. zu ca. 80\% von keiner geeigneten nichtmedikamentösen Therapie profitieren (Alzheimer Vereinigung 2010) [3]. Zudem wird das zahnmedizinische Team nach Diagnosestellung in aller Regel bei der Etablierung eines langfristigen Therapie- und Betreuungskonzeptes nicht einbezogen. Andererseits muss erkannt werden, dass die meisten zahnärztlichen Fachpersonen mit ihren Mitarbeitenden auf die (oral-)medizinischen Herausforderungen bei Menschen mit Demenz nicht ausreichend vorbereitet sind und infolgedessen mit der Betreuungssituation überfordert wären. Diese Sachverhalte führen unweigerlich dazu, dass die von Demenz betroffenen Personen, und nicht selten aus Überlastung auch die sie betreuenden Angehörigen, bereits in der häuslichen Betreuungsphase allmählich und häufig unbemerkt aus der regelmässigen oralmedizinischen Betreuung ausscheren. Die daraus resultierenden negativen Folgen für die Mundgesundheit, wie kariöse oder parodontale Schädigung der Dentition, gelangen dann bei den Demenzkranken, wenn überhaupt, erst bei der Institutionalisierung zur Diagnose, also in einer Krankheitsphase, in der in aller Regel nur noch palliative Massnahmen möglich sind $[4,5]$.

\section{Wechselwirkungen zwischen oralen Erkrankungen und Demenz}

Orale Erkrankungen wiederum können negative Folgen für die Gesamtgesundheit und das Wohlbefinden des meist mehrfach erkrankten, also nicht nur an Demenz leidenden Menschen haben. Hierbei ist nicht nur die Auswirkung marginaler und apikaler parodontaler Infekte auf kardio- [6] bzw. zerebrovaskuläre [7] und Atemwegserkrankungen $[8,9]$ sowie ihre wechselseitige Beziehung mit dem Diabetes mellitus Typ 2 von Bedeutung [10]. Auch ein direkter Link zwischen parodontaler bzw. systemischer Entzündung und der Demenz vom AlzheimerTyp wird diskutiert [11-13]. Zusätzlich weisen experimentelle Daten darauf hin, dass eine reduzierte okklusale Abstützung oder insuffizienter Zahnersatz die Gangsicherheit insbesondere bei eingeschränkter Kognition zu beeinträchtigen vermögen $[14,15]$ und möglicherweise eine positive Korrelation zwischen Kauleistung und kognitiver Leistungsfähigkeit besteht [16]. Leider wird häufig auch das Potenzial oraler Beschwerden für die Beeinträchtigung des Verhaltens und Wohlbefindens von an Demenz erkrankten Menschen unterschätzt; deshalb werden entsprechende Zusammenhänge im Praxisalltag gerne übersehen [17].

\section{Früherkennung neurokognitiver Störungen} in der zahnärztlichen Praxis

Aufgrund dieser Sachverhalte und angesichts der stetig wachsenden Zahl von an Demenz erkrankten Menschen ist es an der Zeit, dass auch in der Zahnmedizin die ärztliche Verantwortung wahrgenommen wird. Diese kann sich nicht nur - wie bisher - auf die rein therapeutisch relevanten Aspekte mit allzu oft lediglich palliativem Charakter beschränken, sondern muss in erster Linie der Prävention dienen [18]. Letztere bedingt aufgrund der häufig fehlenden Demenzdiagnose die Fähigkeit, auch in der zahnmedizinischen Praxis kognitive Defizite frühzeitig wahrzunehmen. Dabei muss erkannt werden, dass gerade der Zahnarzt und sein Team für ein solches Screening altersbedingter Erkrankungen wie Demenz besonders geeignet sind, indem sie gegenüber dem (Haus-)Arzt nicht nur den grossen Vorteil haben, ihre Patienten in aller Regel in längeren Konsultationen zu sehen. Die Patienten sind zudem im zahnmedizinischen Kontext einer grösseren Belastungssituation ausgesetzt, die es gerade dem älteren Menschen erschwert, etwaige gesundheitliche Defizite zu kompensieren. Dies trifft in beispielhafter Weise auf kognitive Einschränkungen zu. Ein solches routinemässiges Patientenscreening setzt allerdings ein Training des gesamten zahnmedizinischen Teams im Erkennen insbesondere von frühen Symptomen einer neurokognitiven Beeinträchtigung voraus, die vom Betroffenen zwar noch kompensiert werden können und ihm eine noch unabhängige Lebensführung erlauben, aber trotzdem erste Anzeichen einer Demenz oder Hinweise auf andere Ursachen, wie Nebenwirkungen von Medikamenten oder Malnutrition, sein können. Nicht nur der Zahnarzt mit seiner Dentalassistentin oder Dentalhygienikerin im klinischen Kontext, sondern auch die Sekretärin im administrativen Bereich und Empfang können Alltagssituationen nutzen, um die kognitive Leistungsfähigkeit der älteren Patienten zu testen und Einbussen zu erkennen [19, 20].

\section{Patientenbeobachtung}

$\mathrm{Zu}$ diesem Zweck hat sich im Praxisalltag die Anwendung einer Checkliste zum mehrdimensionalen Patientenscreening (MPS) bewährt, die in kognitiver Hinsicht Testsituationen aus dem zahnmedizinischen Kontext nutzt $[19,20]$. Entsprechend eignet sie sich auch dazu, die sich ändernden Ressourcen kognitiv eingeschränkter Menschen für die oralmedizinische Langzeitbetreuung laufend zu überprüfen, um bei Bedarf rechtzeitig präventive sowie therapeutische Massnahmen anpassen zu können.

Ein weiteres geeignetes Hilfsmittel stellt die Validation nach Naomi Feil dar [21]. Diese Methode erleichtert Angehörigen sowie Fachpersonen die Berücksichtigung der 
aktuellen Situation und Stimmungslage bei der Kommunikation mit in ihrer Orientierung eingeschränkten Menschen.

\section{Checkliste zum mehrdimensionalen Patientenscreening}

Diese unterstützt das zahnmedizinische Team dabei, für die Mundgesundheit relevante Auffälligkeiten oder Veränderungen im Verhalten bzw. Erscheinungsbild alternder Patienten frühzeitig zu erkennen, die in erster Linie Hinweise auf eine mögliche depressive Verstimmung, ein demenzielles Syndrom oder eine Malnutrition, in zweiter Linie aber auch auf andere Erkrankungen und ihre (Poly-)Pharmakotherapie zu geben vermögen. Die Checklisten-gestützte Patientenbeobachtung kann durch das ganze Team im Rahmen der etablierten organisatorischen und therapeutischen Praxisprozesse ohne zusätzlichen Zeitaufwand durchgeführt werden [19, 20].

Als Vorlage der Symptomliste für kognitive Einschränkungen dient zu weiten Teilen der Mini-Mental Status (MMS) nach Folstein et al. [22]. Dieser gilt als gut validiertes Screeningverfahren für Demenz, dessen Einzeltests im Folgenden auf den oralmedizinischen Kontext übertragen zur Darstellung gelangen.

\section{Erscheinungsbild}

Negative Veränderungen in der Kleidung oder in der Körperpflege können Hinweise auf eine Verschlechterung der Lebenssituation geben, die beispielsweise als Folge von Verlusten - wie der Tod des Lebenspartners - auftritt und für die keine ausreichenden Bewältigungsstrategien zur Verfügung stehen. Sie können somit Ausdruck einer Depression sein. Andere altersspezifische Erkrankungen, die das Erscheinungsbild zu beeinträchtigen vermögen, sind Demenzen oder Morbus Parkinson. Eine deutliche Abnahme des Körpergewichtes über kürzere Zeit kann ein Indikator für das Vorhandensein einer oder mehrerer Krankheiten mit Malnutrition als Folge sein.

\section{Verhalten}

- Umständlich, weitschweifig: Angeordnete Handlungen wie z.B. das Wiederholen von Mundhygienemassnahmen werden nicht zielgerichtet ausgeführt und von der eigentlichen Aufgabe ablenkend kommentiert.

- Motorische Unruhe: Hin- und Herschaukeln des Oberkörpers, ständiges Nesteln mit dem Taschentuch in den Händen.

- Reduzierte Aufmerksamkeit: Plötzliche, eventuell fluktuierend auftretende geistige Abwesenheit. Aufmerksamkeit kann nicht auf ein Objekt (Hygieneinstrument), ein Vorgehen (Mundhygieneinstruktion) oder auf Neues gerichtet werden.
- Affektlabil, affektarm, ratlos: Situationsfremde, nicht adäquate Reaktionen im therapeutischen Kontext wie Teilnahmslosigkeit, Gleichgültigkeit, Apathie, aber auch Aggression, eventuell fluktuierend auftretend.

- Verwirrtheit, Angst, Halluzinationen, Wahn: Plötzliches Aufstehen im Wartebereich oder vom Behandlungsstuhl, Herumirren in der Praxis. Die betroffene Person weiss nicht mehr, wo sie sich befindet, will in einem unangebrachten Moment nach Hause gehen, kann übliche und somit bekannte Behandlungsmassnahmen nicht verstehen, zeigt Reaktionen der Angst auf bisher unauffällig bewältigte Behandlungssituationen. Es treten unerwartete, der Situation nicht entsprechende Gefühle des Bedrohtwerdens auf, eventuell verbunden mit Abwehrreaktionen.

- Plötzliche Veränderungen: Unerwartet, eventuell fluktuierend auftretende, nicht situationsgerechte Reaktionen.

\section{Zeitliche Orientierung [22]}

- Datum, Wochentag, Monat, Jahr: Der Patient ist nicht in der Lage, beim Eintrag in die die Krankengeschichte auf die Frage «Welches Datum haben wir schon wieder heute?» korrekt zu antworten.

- Zeitverschiebung: Der Patient erscheint fälschlicherweise an einem anderen Tag oder zu einer anderen Uhrzeit als vereinbart, ist aber überzeugt, den Termin korrekt eingehalten zu haben.

- Terminwahrnehmung, Pünktlichkeit: Termine gehen trotz kurzfristiger telefonischer Erinnerung wiederholt vergessen bzw. der Patient erscheint regelmässig zu früh oder zu spät. Achten Sie bei der Begründung für das Fehlverhalten auf Zeichen der Überforderung. - Fähigkeit der Terminplanung: Der Vorgang der Terminvereinbarung oder die Festlegung des Nachsorgeintervalls sind beeinträchtigt bzw. umständlich oder werden an Begleitpersonen delegiert (Vermeidungsverhalten). Früher benutzte elektronische Planungssysteme werden plötzlich nicht mehr angewendet und an ihrer Stelle ein Terminkärtchen verlangt.

\section{Räumliche Orientierung [22]}

- An- und Rückreiseweg, Stockwerk, Praxis: Der Anoder Rückreiseweg inklusive benutzte Verkehrsmittel und zurückgelegte Route können nicht mehr im Detail beschrieben werden, der momentane Aufenthaltsort wird nicht mehr erkannt, bekannte Örtlichkeiten in der Praxis wie die Toilette oder der Empfangsbereich werden nicht mehr auf Anhieb gefunden.

- Durchführung von Mundhygienemassnahmen: Das räumliche Verständnis für die Planung und Durchführung von Hygienemassnahmen ist deutlich eingeschränkt oder nicht mehr gegeben. 


\section{Gedächtnis [22]}

Merkfähigkeit, Hygieneinstruktionen: Es hat sich bewährt, Aufklärungen über geplante Therapien und Eingriffe sowie Hygieneinstruktionen zu Beginn einer Sitzung durchzuführen und an deren Ende kurz wiederholen zu lassen. Dieses Vorgehen erlaubt die Überprüfung der Merkfähigkeit. Dabei steht nicht die Wiedergabefähigkeit aller Details im Vordergrund; diese kann auch bei jüngeren Menschen z.B. infolge von Interesselosigkeit beeinträchtigt sein. Vielmehr geht es darum, ob die erklärten Vorgänge als Ganzes korrekt und logisch rekapituliert und neu instruierte Hygieneinstrumente korrekt verstanden und wiedergegeben werden.

\section{Erkennen, Verständnis [22]}

Erkennen und Anwenden von Gebrauchsgegenständen: Die Funktion bekannter bzw. neu instruierter Hygieneinstrumente oder Terminkärtchen wird nicht mehr verstanden. Alltägliche Verrichtungen wie Spülen des Mundes und Handhabung des eigenen Zahnersatzes oder bisher beherrschte Mundhygienemassnahmen können nach Aufforderung nicht mehr korrekt ausgeführt werden.

\section{Exekutive Kognition}

- Planung/Durchführung komplexer Prozesse: Mundhygienemassnahmen oder Terminvereinbarungen können nicht mehr einwandfrei geplant und durchgeführt werden. Zur Testung der exekutiven Kognition hat sich der Uhrtest [23] bewährt, um Ressourcen wie Lern- und Adaptationsfähigkeit an eine zu verändernde intraorale Situation besser einschätzen zu können. Die Fähigkeit, falsch herum gereichten Zahnersatz reflexartig korrekt einzugliedern, testet ebenfalls die exekutive Kognition älterer Menschen [20].

- Abstimmung der Aufmerksamkeitsressourcen: Die Aufmerksamkeit kann nicht mehr gleichzeitig auf die Dentalhygienikerin, die beispielsweise den Nachvollzug einer bestimmten Mundhygienemassnahme anordnet, und auf die Koordination der eigenen kognitiven sowie motorischen Vorgänge gerichtet werden.

\section{Multitasking [22]}

Gleichzeitige Ausführung mehrerer Aufgaben: Ältere Menschen sollen auf dem Weg vom Wartebereich in das Behandlungszimmer in ein Gespräch verwickelt werden. Wird der Gang beim Beantworten von Fragen langsamer bzw. unsicherer oder bleibt die Person dabei sogar stehen, so ist das Multitasking eingeschränkt.

\section{Gangsicherheit [22]}

Gangvariabilität und Gleichgewichtsstörungen können sowohl Folge einer Erkrankung (z.B. Demenz) als auch einer medikamentösen Therapie sein. Gemeinsam mit einer beeinträchtigten Beweglichkeit können sie die Mobilität und somit die Recallfähigkeit deutlich einschränken.

\section{Sprachfähigkeit [22]}

Satz- und Wortbildung sind erschwert bzw. Worte werden sinnfremd verwendet.

\section{Lesefähigkeit [22]}

Die Abgabe von Merkblättern oder handschriftlichen Notizen, z.B. zu Mundhygieneinstruktionen, sind sinnvoll. Dabei sollte aber auch die Lesefähigkeit überprüft werden - z.B. mit folgender Frage: «Bitte lesen Sie mir die oberste Zeile vor, damit ich sicher bin, dass die vorliegende Schriftgrösse bzw. meine Handschrift für Sie lesbar sind.» Achten Sie darauf, ob und mit welcher Begründung versucht wird, die Ausführung dieses Auftrags zu vermeiden bzw. an eine Begleitperson zu delegieren (Vermeidungsverhalten). Insistieren Sie auf der Ausführung des Auftrags, indem Sie beispielsweise dem Patienten die Gelegenheit geben, seine Lesehilfe aufzusetzen.

\section{Schreibfähigkeit [22]}

Lassen Sie ältere Menschen Notizen zu Informationen machen oder das Terminkärtchen unter Aufsicht selbst ausfüllen. Achten Sie auch hier auf korrekte Ausführung und ein mögliches Vermeidungsverhalten.

\section{Begleitung}

Achten Sie auf den Grad der Selbstständigkeit bei der Bewältigung der alltäglichen Aktivitäten, um beispielsweise die Recall- und Hygienefähigkeit älterer Menschen besser einschätzen zu können.

\section{Soziales Umfeld}

Soziale Veränderungen wie der Verlust des Lebenspartners durch Tod oder Scheidung bzw. der Selbstständigkeit (z.B. Hilfs- und Betreuungsbedarf, Übertritt in ein Heim) sollen aufmerksam verfolgt werden, da diese vielseitige Folgen auf die psychische und physische Gesundheit älterer Menschen haben können und somit auch die oralmedizinische Betreuungsfähigkeit zu beeinträchtigen vermögen. 


\section{Validation}

Die Validation nach Feil [22] basiert ebenfalls auf einer systematischen und genauen Beobachtung des Verhaltens älterer Menschen mit kognitiven Einschränkungen. Diese Methode unterstützt Angehörige und Fachpersonen dabei, einen positiven Zugang zu diesen Patienten zu finden, indem das Verständnis ihrer aktuellen Situation bzw. Stimmungslage sowie die Wahl geeigneter verbaler und insbesondere nonverbaler Kommunikationswege gefördert wird.

Das Verhalten von mangelhaft orientierten älteren Menschen kann gemäss Feil [22] durch eines oder mehrere der folgenden Grundbedürfnisse beeinflusst sein:

- Aufarbeiten unerledigter Angelegenheiten;

- Leben in Frieden und Geborgenheit;

- Wohlbefinden und vertraute Beziehungen;

- Wahrung oder Wiederfinden des inneren Gleichgewichts;

- Sinnfindung für die sich verändernde Wirklichkeit;

- Anerkennung (Status, Identität und Selbstwertgefühl), Respekt und Ich-Stärkung;

- soziale Eingebundenheit;

- Ausdruck und Wahrnehmung der eigenen Gefühle;

- Liebe, Fürsorge und Freiheit;

- Bedürfnis von Nähe oder Distanz;

- Linderung von Schmerzen oder Unannehmlichkeiten. Die Bewältigung der zum gegebenen Zeitpunkt betroffenen Bedürfnisse kann dazu führen, dass die Patienten sich in erschwert erreichbare Räume bzw. Wirklichkeiten zurückziehen oder in unkonventioneller Weise Hilfe suchen. Das Erkennen dieser Situationen und der damit verbundenen Stimmungslage bedingt eine intensive Beobachtung von Erscheinungsbild und Verhalten. Die folgenden Kriterien können dabei sehr hilfreich sein [22]:

- Haar: ordentlich gekämmt oder wirr;

- Augen: weit aufgerissen, verkniffen, geschlossen, trocken oder feucht, achtsamer oder abwesender Blick;

- Stirn: Furchen, Augenbrauen nach oben gezogen oder nach unten hängend;

- Nase: schmal oder bebende Nasenflügel;

- Kieferhaltung: angespannt oder locker;

- Lippen/Mund: verkniffen oder entspannt, Schmollmund, Lächeln, hängende Mundwinkel;

- Schultern: hochgezogen oder hängend, nach vorne oder hinten gerichtet, zusammengesunken;

- Brust: Atmung schnell oder langsam, in den Brustkorb oder in den Bauch;

- Körperhaltung: angespannt oder locker, aufrecht oder vornübergebeugt bzw. zusammengesunken, offen oder abwehrend;

- Arme und Hände: angespannt oder entspannt, in Bewegung oder in Ruhe;
- Beine und Füsse: angespannt oder entspannt, geöffnet oder eng gestellt bzw. überschlagen, angezogen oder ausgestreckt;

- räumliche Bewegungen: gezielt oder ungezielt, harmonisch oder steif, normal, verzögert oder heftig.

Das Erkennen der aktuellen Situation und Stimmungslage bildet den Schlüssel zu einer zielgerichteten Mobilisierung von Ressourcen und somit zu einer verbesserten Kommunikation sowie Kooperation mit dem an kognitiver Einschränkung leidenden älteren Menschen. Das nachfolgende Situationsbeispiel veranschaulicht zum Schluss die zielgerichtete klinische Anwendung der Checkliste und der Validation. Weitere Empfehlungen für den Umgang und die Kommunikation mit demenzkranken Menschen wurden bereits in einer unserer früheren Arbeiten veröffentlicht [1] und in einem Merkblatt der Schweizerischen Alzheimervereinigung zusammengefasst [24].

\section{Situationsbeispiel}

Gerade bei älteren Menschen mit einer komplexer werdenden medizinischen und psychosozialen Lebenssituation kann es gehäuft zu einer Verweigerung von oralmedizinischen Interventionen kommen. Tritt diese vor Abschluss eines invasiven Eingriffs auf, indem der Patient plötzlich den Schwebetisch wegschiebt und mit den Worten «Ich will nach Hause» aufsteht, so wird das Behandlungsteam vor eine nicht immer leicht zu lösende Herausforderung gestellt. In einer solchen Situation kann es natürlich hilfreich sein, eine aktualisierte medizinische Anamnese und eine gültige Liste der gegenwärtig eingenommenen Medikamente vorliegen zu haben, um eine ursächliche Erklärung für dieses Verhalten zu finden. Doch häufig reicht dieses Wissen nicht aus, da gerade bei Demenz der Grossteil der Betroffenen ohne Diagnose lebt und somit keine entsprechenden Angaben machen kann. Liegt eine kognitive Beeinträchtigung für das beschriebene, auf eine akute Verwirrtheit (Delir) hinweisende Verhalten vor, so ist ein Einreden auf den Patienten oder ein Beschwichtigen in der Regel nicht zielführend.

Eine systematische, Checklisten-gestützte Beobachtung älterer Patienten über die Zeit bietet dagegen eine höhere Wahrscheinlichkeit, im Voraus Hinweise auf allmählich auftretende Defizite zu gewinnen, die einen Einfluss auf die Indikationsstellung für eine bestimmte Intervention und deren Vorbereitung haben können. Liegen Beobachtungen in Richtung einer kognitiven Einschränkung vor, so könnte die Begleitung durch eine dem Patienten vertraute Person hilfreich sein, die auch während des Eingriffs im Behandlungsraum anwesend ist. Zudem kann durch die Sicherstellung einer guten Nahrungs- sowie Flüssigkeitsaufnahme vor einer Intervention ohne 
Narkose dem Auftreten eines akuten Verwirrtheitszustandes entgegengewirkt werden. Schliesslich wäre das Behandlungsteam durch entsprechende Beobachtungen vorgewarnt und somit auf einen solchen Zwischenfall besser vorbereitet.

Die Validation vor sowie während des Eingriffs erleichtert es dem Zahnarzt und seiner Assistenz, die Stimmungslage des Patienten richtig einzuschätzen und verständnisvoll zu spiegeln, um sie in der Folge lösungsorientiert modulieren zu können. Dabei muss man sich bewusst sein, dass die ersten Anzeichen von z.B. Angst oder Ärger als mögliche Ursachen für das besagte Verhalten bei Menschen mit Demenz mehrdeutig und subtil sein können, sodass sie bei fehlender Validation leicht übersehen werden und entsprechend ein situationsgerechtes Verhalten nicht mehr gewährleistet ist.

Eine mögliche Auflösung dieser schwierigen Behandlungssituation könnte darin bestehen, den Wunsch nach Hause gehen zu wollen umzudeuten: «Ich sehe, Sie möchten lieber spazieren gehen. Kommen Sie, drehen wir doch gemeinsam eine gemütliche Runde durch die Praxis. Und Ihre Begleitung kommt gleich mit.» Hiermit wird das Verhalten des Patienten in einen neuen, positiven Rahmen gestellt, der auch die Gelegenheit bietet, während dem Rundgang an einem geeigneten Ort etwas Wasser oder Tee anzubieten. Sollten es Gesundheitszustand und die intraorale Situation erlauben, kann dem Getränk noch Glukoselösung aus Trinkampullen hinzugefügt werden. Auf diese Weise wird gleichzeitig die Versorgung mit Flüssigkeit und Energie verbessert. Der positive Rahmen kann weiter verstärkt werden, indem der Behandler selbst ein Glas Wasser zu sich nimmt und somit eine aus dem Alltag vertraute Situation schafft, die eine noch bessere Grundlage bietet, den Weg zurück zur Behandlung zu finden.

\section{Disclosure Statement}

Die Autoren bestätigen, dass im Zusammenhang mit dieser Arbeit keine Interessenkonflikte bestehen.

\section{Literatur}

1 Besimo CE, Besimo-Meyer RH: Wenn Worte ihre Bedeutung verlieren. Kommunikation mit an Demenz erkrankten Menschen. Participation 2014;3:14-16.

2 Streffer J: Das gesunde und das kranke Gehirn von Hochaltrigen - Neurobiologie des Gehirns im hohen Alter; in Petzold HG, Horn E, Müller L (Hrsg): Hochaltrigkeit. Herausforderung für persönliche Lebensführung und biopsychosoziale Arbeit. Wiesbaden, VS Verlag für Sozialwissenschaften, 2011, pp 77-90.

3 Schweizerische Alzheimervereinigung: 107500 Menschen mit Demenz in der Schweiz. Schweizerische Alzheimervereinigung, 2012.

4 Besimo CE, Besimo-Meyer RH: Orale Gesundheit von Menschen mit Demenz. Schweiz Z Ganzheitsmed 2015;27:44-49.

5 Adam H, Preston AJ: The oral health of individuals with dementia in nursing homes. Gerodontology 2006;23:99-105.

6 Mattila KJ, Pussinen PJ, Paju S: Dental infections and cardiovascular diseases: a review. J Periodontol 2005;76:2085-2088.

7 Ghizoni JS, Taveira LA, Garlet GP, Ghizoni MF, Pereira JR, Dionisio TJ, Brozoski DT, Santos CF, Sant'Ana AC: Increased levels of Porphyromonas gingivalis are associated with ischemic and hemorrhagic cerebrovascular disease in humans: an in vivo study. J Appl Oral Sci 2012;20:104-112.

8 Scannapieco FA: Orale Erkrankungen und Infektionen der Atemwege. Acta Med Dent Helv 2000;5:74-77.

9 Yoneyama T, Yoshida M, Ohrui T, Mukaiyama H, Okamoto H, Hoshiba K, Ihara S, Yanagisawa S, Ariumi S, Morita T, Mizuno Y Ohsawa T, Akagawa Y, Hashimoto K, Sasaki $\mathrm{H}$ : Oral care reduces pneumonia in older patients in nursing homes. J Am Geriatr Soc 2002;50:430-433.
10 Soskolne WA, Klinger A: The relationship between periodontal diseases and diabetes: an overview. Ann Periodontol 2001;6:91-98.

-11 Kamer AR, Craig RG, Dasanayake AP, Brys M, Glodzik-Sobanska L, de Leon MJ: Inflammation and Alzheimer's disease: possible role of periodontal diseases. Alzheimers Dement 2008;4:242-250.

12 Watts A, Crimmins EM, Gatz M: Inflammation as a potential mediator for the association between periodontal disease and Alzheimer's disease. Neuropsychiatr Dis Treat 2008; 4:865-876

13 Sparks Stein P, Steffen MJ, Smith C, Jicha G, Ebersole JL, Abner E, Dawson D: Serum antibodies to periodontal pathogens are a risk factor for Alzheimer's disease. Alzheimers Dement 2012;8:196-203.

14 Kato T, Usami T, Noda Y, Hasegawa M, Ueda M, Nabeshima T: The effect of the loss of molar teeth on spatial memory and acetylcholine release from the parietal cortex in aged rats. Behav Brain Res 1997;83:239-242.

15 Yoshida M, Morikawa H, Kanehisa Y, Taji T, Tsuga K, Akagawa Y: Functional dental occlusion may prevent falls in elderly individuals with dementia. J Am Geriatr Soc 2005;53: 1631-1632.

16 Banu RF, Veeravalli PT, Kumar VA: Comparative evaluation of changes in brain activity and cognitive function of edentulous patients, with dentures and two-implant supported mandibular overdentures - pilot study. Clin Implant Dent Relat Res 2015;DOI: 10.1111/cid.12336.

17 Shimazaki Y: Influence of dentition status on physical disability, mental impairment, and mortality in institutionalized elderly people. J Dent Res 2001;80:340-345.
18 Besimo CE: Paradigmenwechsel zugunsten einer besseren oralen Gesundheit im Alter. Swiss Dent J 2015;125:599-604.

19 Besimo CE: Mehrdimensionale Diagnostik. Medizinisch-psychosoziales Screening des alternden Menschen in der zahnärztlichen Praxis; in Besimo C (Hrsg): Zahnärztliche Betreuung älterer Menschen, Schriftenreihe, Heft I. Zürich, Springer Medizin Schweiz, 2009.

20 Besimo CE: Mehrdimensionales Assessment des alternden Menschen in der zahnärztlichen Praxis. Medizinische Screeninginstrumente. Bern, Schweizerische Zahnärzte-Gesellschaft SSO, 2015.

21 de Klerk-Rubin V: Mit dementen Menschen richtig umgehen. Validation für Angehörige, ed 2. München, Ernst Reinhardt, 2009.

22 Folstein MF, Folstein SE, Mc Hugh PR: 'Mini-mental state': a practical method for grading the cognitive state of patients for the clinician. J Psychiatr Res 1975;12:189-198.

23 Shulman KI: Clock-drawing: is it the ideal cognitive screening test? Int J Geriatr Psychiatry $2000 ; 15: 548-561$.

24 Schweizerische Alzheimervereinigung: Demenzkranke Menschen als Kunden. Tipps für den Umgang. Menschen mit Demenz als Patienten: In der zahnmedizinischen Praxis. Schweizerische Alzheimervereinigung, 2015. 